\title{
Generalized Schrödinger Equations and Jordan Pairs
}

\section{S. I. Svinolupov}

Institute of Mathematics, Ural Branch of the Academy of Sciences of USSR, ul. Chernyshevskiy 112, SU-450000 Ufa, USSR

\begin{abstract}
The criteria of integrability for the nonlinear Schrödinger-type systems are obtained. One-to-one correspondence between such integrable systems and the Jordan pairs is established. It turns out that irreducible systems correspond to simple Jordan pairs. An infinite series of generalized symmetries and local conservation laws for such systems are completely described.
\end{abstract}

\section{Introduction}

Let us consider a nonlinear system of $(N+M)$ equations:

$$
\begin{aligned}
& \mathbf{u}_{t}^{i}=\mathbf{u}_{x x}^{i}+\mathbf{a}_{j k m}^{i} \mathbf{u}^{j} \mathbf{v}^{k} \mathbf{u}^{m}, \quad i=1, \ldots, N, \\
& \mathbf{v}_{t}^{i}=-\mathbf{v}_{x x}^{i}-\tilde{\mathbf{a}}_{j k m}^{i} \mathbf{v}^{j} \mathbf{u}^{k} \mathbf{v}^{m}, \quad i=1, \ldots, M,
\end{aligned}
$$

where $\mathbf{u}^{i}, \mathbf{v}^{i}$ depend on $t, x ; \mathbf{a}_{j k m}^{i}, \tilde{\mathbf{a}}_{j k m}^{i}$ are constants which may be assumed such that

$$
\mathbf{a}_{j k m}^{i}=\mathbf{a}_{m k j}^{i}, \quad \tilde{\mathbf{a}}_{j k m}^{i}=\tilde{\mathbf{a}}_{m k j}^{i},
$$

without loss of generality. The number of $\mathbf{u}^{i}$ 's and $\mathbf{v}^{i}$ 's may vary. In (0.1) and everywhere below the summation on repeated indices is assumed.

In particular among the systems of the form (0.1) there is the system

$$
\mathbf{u}_{t}=\mathbf{u}_{x x}+\mathbf{u}^{2} \mathbf{v}, \quad \mathbf{v}_{t}=-\mathbf{v}_{x x}-\mathbf{v}^{2} \mathbf{u},
$$

which may be reduced to the well-known nonlinear Schrödinger equation

$$
i \mathbf{z}_{t}=\mathbf{z}_{x x}+\mathbf{z}|\mathbf{z}|^{2}, \quad \mathbf{z}=\mathbf{z}(t, x) .
$$

The system (0.3) possesses an infinite series of generalized symmetries and local conservation laws (see, for example, [1]). We'll call the systems $(0.1)$ which have similar properties the generalized Schrödinger equations. There are examples of such systems different from (0.3) (see [2-4]). A wide class of generalized Schrödinger equations is found in [4] by means of algebraic construction which 
assumes that the system possesses an (L-A)-pair of a special form. This construction associates with each irreducible Hermitian symmetric space some system of the form (0.1).

The goals of the paper are 1) to find the conditions on $\mathbf{a}_{j k m}^{i}, \tilde{\mathbf{a}}_{j k m}^{i}$ for (0.1) to provide generalized symmetries or local conservation laws; 2) to describe constructively all such remarkable systems. In contrast with [4] we don't make initially any additional assumptions on the algebraic nature of the systems considered.

The existence of generalized symmetries or local conservation laws yield necessary and sufficient conditions which are found below. The conditions are the set of polynomial relations on $\mathbf{a}_{j k m}^{i}, \tilde{\mathbf{a}}_{j k m}^{i}$, which one can easily check for every particular system by means of computer algebra. For systems satisfying these conditions the formal recursion operator is constructed.

The one-to-one correspondence between systems $(0.1)$ having at least one nondegenerate generalized symmetry or nondegenerate conservation law and Jordan pairs (see [5]) is established. Moreover, we show that the most interesting "non-triangle" systems correspond to simple Jordan pairs. An infinite series of generalized symmetries and local conservation laws for such systems are completely described.

\section{The Algebraic Interpretation of Systems of the (0.1) Type. Jordan Pairs}

It is naturally to expect that the system $(0.1)$ has generalized symmetries or local conservation laws only if $\mathbf{a}_{j k m}^{i}, \tilde{\mathbf{a}}_{j k m}^{i}$ satisfy some algebraic equations. It is convenient to choose right now the appropriate algebraic objects in terms of which the conditions on constants are formulated in the most simple and clear way. Let $\mathbf{V}$ and $\tilde{\mathbf{V}}$ be two vector spaces over $\mathbb{C}$ of dimensions $N$ and $M$, respectively. We define the trilinear multiplications $\mathbf{T}$ and $\widetilde{\mathbf{T}}$ :

$$
\begin{array}{ll}
\mathbf{V} \times \tilde{\mathbf{V}} \times \mathbf{V} \rightarrow \mathbf{V}, & \mathbf{T}(\mathbf{x}, \tilde{\mathbf{y}}, \mathbf{z})=\{\mathbf{x} \tilde{\mathbf{y}} \mathbf{z}, \\
\tilde{\mathbf{V}} \times \mathbf{V} \times \tilde{\mathbf{V}} \rightarrow \tilde{\mathbf{V}}, & \tilde{\mathbf{T}}(\tilde{\mathbf{x}}, \mathbf{y}, \tilde{\mathbf{z}})=\{\tilde{\mathbf{x}} \mathbf{y} \tilde{\mathbf{z}}\},
\end{array}
$$

We assume for any $\mathbf{x}, \mathbf{y} \in \mathbf{V}, \tilde{\mathbf{x}}, \tilde{\mathbf{y}} \in \tilde{\mathbf{V}}$ the identities

$$
\{\mathbf{x} \tilde{\mathbf{x}} \mathbf{y}\}=\{\mathbf{y} \tilde{\mathbf{x}} \mathbf{x}\}, \quad\{\tilde{\mathbf{x}} \mathbf{x} \tilde{\mathbf{y}}\}=\{\tilde{\mathbf{y}} \mathbf{x} \tilde{\mathbf{x}}\} .
$$

to hold. We call the above algebraic object a "pair of vector spaces with triple multiplication" and denote it by $(\mathbf{V}, \tilde{\mathbf{V}})$.

Let $\mathbf{e}_{1}, \mathbf{e}_{2}, \ldots, \mathbf{e}_{N}$ and $\tilde{\mathbf{e}}_{1}, \tilde{\mathbf{e}}_{2}, \ldots, \tilde{\mathbf{e}}_{M}$ be bases of $\mathbf{V}$ and $\tilde{\mathbf{V}}$, respectively. Then the multiplication is given by

$$
\left\{\mathbf{e}_{j} \tilde{\mathbf{e}}_{k} \mathbf{e}_{m}\right\}=\mathbf{a}_{j k m}^{i} \mathbf{e}_{i}, \quad\left\{\tilde{\mathbf{e}}_{j} \mathbf{e}_{k} \tilde{\mathbf{e}}_{m}\right\}=\tilde{\mathbf{a}}_{j k m}^{i} \tilde{\mathbf{e}}_{i} .
$$

It follows from (1.2) that the structure constants $\mathbf{a}_{j k m}^{i}, \tilde{\mathbf{a}}_{j k m}^{i}$ of the multiplication are symmetric with respect to the first and the third subscripts.

Let us establish one-to-one correspondence between the pairs $(\mathbf{V}, \tilde{\mathbf{V}})$ and the systems $(0.1)$ by means of identifying the structure constants with the constants from the right-hand side of a system. This correspondence is correctly defined. Indeed the form of system (0.1) is invariant with respect to the transformations

$$
\mathbf{u}^{i}=\mathbf{J}_{j}^{i} \mathbf{U}^{j}, \quad \mathbf{v}^{i}=\widetilde{\mathbf{J}}_{j}^{i} \mathbf{V}^{j},
$$


where $\mathbf{J}$ and $\widetilde{\mathbf{J}}$ are arbitrary nondegenerate constant $N \times N$ and $M \times M$ matrices. In more detail the result of applying the transformation (1.4) to (0.1) is the system

$$
\begin{array}{ll}
\mathbf{U}_{t}^{i}=\mathbf{U}_{x x}^{i}+\mathbf{A}_{j k m}^{i} \mathbf{U}^{j} \mathbf{V}^{k} \mathbf{U}^{m}, & i=1,2, \ldots, N, \\
\mathbf{V}_{t}^{i}=-\mathbf{V}_{x x}^{i}-\tilde{\mathbf{A}}_{j k m}^{i} \mathbf{V}^{j} \mathbf{U}^{k} \mathbf{V}^{m}, & i=1,2, \ldots, M,
\end{array}
$$

where the constants $\mathbf{A}_{j k m}^{i}, \tilde{\mathbf{A}}_{j k m}^{i}$ are defined by the formulas

$$
\begin{aligned}
& \mathbf{A}_{j k m}^{i}=\left(\mathbf{J}^{-1}\right)_{r}^{i} \mathbf{a}_{n s p}^{r} \mathbf{J}_{j}^{n} \widetilde{\mathbf{J}}_{k}^{s} \mathbf{J}_{m}^{p}, \\
& \tilde{\mathbf{A}}_{j k m}^{i}=\left(\tilde{\mathbf{J}}^{-1}\right)_{r}^{i} \tilde{\mathbf{a}}_{n s p}^{r} \tilde{\mathbf{J}}_{j}^{n} \mathbf{J}_{k}^{s} \widetilde{\mathbf{J}}_{m}^{p} .
\end{aligned}
$$

Two systems connected by the transformation (1.4) will be called equivalent. From (1.6) it follows that in terms of the corresponding pair $(\mathbf{V}, \widetilde{\mathbf{V}})$ the transformation (1.4) of the system (0.1) is simply a changing of the basis $\mathbf{E}_{i}=\mathbf{J}_{i}^{j} \mathbf{e}_{j}, \widetilde{\mathbf{E}}_{i}=\widetilde{\mathbf{J}}_{i}^{j} \tilde{\mathbf{e}}_{j}$.

It may happen that as the result of the transformation (1.4) we obtain a "triangle" system (1.5), i.e. a system with subsystem of the form

$$
\begin{array}{ll}
\mathbf{U}_{t}^{i}=\mathbf{U}_{x x}^{i}+\mathbf{A}_{j k m}^{i} \mathbf{U}^{j} \mathbf{V}^{k} \mathbf{U}^{m}, & i=1,2, \ldots, N_{1}, \\
\mathbf{V}_{t}^{i}=-\mathbf{V}_{x x}^{i}-\tilde{\mathbf{A}}_{j k m}^{i} \mathbf{V}^{j} \mathbf{U}^{k} \mathbf{V}^{m}, & i=1,2, \ldots, M_{1},
\end{array}
$$

where $N_{1}+M_{1}<N+M$. If some transformation (1.4) bringing the system (0.1) to the form (1.7) exists, (0.1) will be called a reducible system, in the opposite case, a nonreducible one. A completely reducible system is the reducible one which is equivalent to a "splitted" system of the form

$$
\begin{aligned}
\mathbf{U}_{t}^{i} & =\mathbf{U}_{x x}^{i}+\mathbf{A}_{j k m}^{i} \mathbf{U}^{j} \mathbf{V}^{k} \mathbf{U}^{m}, & \mathbf{V}_{t}^{i} & =-\mathbf{V}_{x x}^{i}-\tilde{\mathbf{A}}_{j k m}^{i} \mathbf{V}^{j} \mathbf{U}^{k} \mathbf{V}^{m}, \\
\mathbf{W}_{t}^{i} & =\mathbf{W}_{x x}^{i}+\alpha_{j k m}^{i} \mathbf{W}^{j} \mathbf{Z}^{k} \mathbf{W}^{m}, & & \mathbf{Z}_{t}^{i}=-\mathbf{Z}_{x x}^{i}-\tilde{\alpha}_{j k m}^{i} \mathbf{Z}^{j} \mathbf{W}^{k} \mathbf{Z}^{m}
\end{aligned}
$$

(for the sake of uniform notation we denote $\mathbf{U}^{i+N_{1}}=\mathbf{W}^{i}$ if $i=1, \ldots, N-N_{1}$ and $\mathbf{V}^{i+N_{1}}=\mathbf{Z}^{i}$ if $\left.i=1, \ldots, M-M_{1}\right)$.

The described correspondence between the systems $(0.1)$ and the pairs of vector spaces with multiplication allows us to reformulate the concepts of nonreducibility, reducibility, and complete reducibility in the invariant terms. To do this we recall the definition of an ideal. Let $(\mathbf{V}, \tilde{\mathbf{V}})$ be the pair of vector spaces with multiplication, $\mathbf{W}$ and $\tilde{\mathbf{W}}$ are the linear subspaces of $\mathbf{V}$ and $\tilde{\mathbf{V}}$. The pair $(\mathbf{W}, \tilde{\mathbf{W}})$ is called an ideal of the pair $(\mathbf{V}, \tilde{\mathbf{V}})$ if for any elements $\mathbf{x}, \mathbf{y} \in \mathbf{V}, \tilde{\mathbf{x}}, \tilde{\mathbf{y}} \in \tilde{\mathbf{V}}, \mathbf{w} \in \mathbf{W}, \tilde{\mathbf{w}} \in \tilde{\mathbf{W}}$ the conditions

$$
\{\mathbf{w} \tilde{\mathbf{x}} \mathbf{y}\} \in \mathbf{W}, \quad\{\mathbf{x} \tilde{\mathbf{w}} \mathbf{y}\} \in \mathbf{W}, \quad\{\tilde{\mathbf{w}} \mathbf{x} \tilde{\mathbf{y}}\} \in \tilde{\mathbf{W}}, \quad\{\tilde{\mathbf{x}} \mathbf{w} \tilde{\mathbf{y}}\} \in \tilde{\mathbf{W}}
$$

hold. We shall call a pair of vector spaces $(\mathbf{V}, \tilde{\mathbf{V}})$ without ideals the simple one. It's easy to prove the next statement.

Proposition 1.1. For the system (0.1) to be
a) irreducible,
b) reducible,
c) completely reducible,

it's necessary and sufficient that the respective pair of vector spaces with multiplication
a) is simple,
b) has nontrivial ideal,
c) is the direct sum of its ideals. 
It is natural to expect that the algebraic properties of $(0.1)$ such as the existence of symmetries, conservation laws and so on are completely defined through the structure of the corresponding pair of vector space $(\mathbf{V}, \tilde{\mathbf{V}})$. Let us demonstrate this by an example. Consider the nonlinear Schrödinger equation. It possesses the third order symmetry

$$
\mathbf{u}_{\tau_{3}}=\mathbf{u}_{x x x}+3 \mathbf{u v u} \mathbf{u}_{x}, \quad \mathbf{v}_{\tau_{3}}=\mathbf{v}_{x x x}+3 \mathbf{v u v _ { x }} .
$$

By straightforward computations one shows that the system (0.1) has a symmetry of the form

$$
\begin{aligned}
& \mathbf{u}_{\tau_{3}}^{i}=\mathbf{u}_{x x x}^{i}+3 \mathbf{a}_{j k m}^{i} \mathbf{u}^{j} \mathbf{v}^{k} \mathbf{u}_{x}^{m}, \\
& \mathbf{v}_{\tau_{3}}^{i}=\mathbf{v}_{x x x}^{i}+3 \tilde{\mathbf{a}}_{j k m}^{i} \mathbf{v}^{j} \mathbf{u}^{k} \mathbf{v}_{x}^{m},
\end{aligned}
$$

if and only if the constants $\mathbf{a}_{j k m}^{i}, \tilde{\mathbf{a}}_{j k m}^{i}$ satisfy the following constraints

$$
\begin{aligned}
& \mathbf{a}_{j k n}^{i} \mathbf{a}_{m s p}^{n}-\mathbf{a}_{m s n}^{i} \mathbf{a}_{j k p}^{n}-\mathbf{a}_{n s p}^{i} \mathbf{a}_{j k m}^{n}+\mathbf{a}_{m n p}^{i} \tilde{\mathbf{a}}_{k j s}^{n}=0, \\
& \tilde{\mathbf{a}}_{j k n}^{i} \tilde{\mathbf{a}}_{m s p}^{n}-\tilde{\mathbf{a}}_{m s n}^{i} \tilde{\mathbf{a}}_{j k p}^{n}-\tilde{\mathbf{a}}_{n s p}^{i} \tilde{\mathbf{a}}_{j k m}^{n}+\tilde{\mathbf{a}}_{m n p}^{i} \mathbf{a}_{k j s}^{n}=0 .
\end{aligned}
$$

These conditions mean that for any elements $\mathbf{x}, \mathbf{y}, \mathbf{z} \in \mathbf{V}, \tilde{\mathbf{x}}, \tilde{\mathbf{y}}, \tilde{\mathbf{z}} \in \tilde{\mathbf{V}}$ the identities

$$
\begin{aligned}
& \{\mathbf{x} \tilde{\mathbf{y}}\{\mathbf{y} \tilde{\mathbf{x}} \mathbf{z}\}\}-\{\mathbf{y} \tilde{\mathbf{x}}\{\mathbf{x} \tilde{\mathbf{y}} \mathbf{z}\}\}-\{\mathbf{z} \tilde{\mathbf{x}}\{\mathbf{x} \tilde{\mathbf{y}} \mathbf{y}\}\}+\{\mathbf{y}\{\tilde{\mathbf{y}} \mathbf{x} \tilde{\mathbf{x}}\} \mathbf{z}\}=0, \\
& \{\tilde{\mathbf{x}} \mathbf{y}\{\tilde{\mathbf{y}} \mathbf{x} \tilde{\mathbf{z}}\}\}-\{\tilde{\mathbf{y}} \mathbf{x}\{\tilde{\mathbf{x}} \mathbf{y} \tilde{\mathbf{z}}\}\}-\{\tilde{\mathbf{z}} \mathbf{x}\{\tilde{\mathbf{x}} \mathbf{y} \tilde{\mathbf{y}}\}\}+\{\tilde{\mathbf{y}}\{\mathbf{y} \tilde{\mathbf{x}} \mathbf{x}\} \tilde{\mathbf{z}}\}=0 .
\end{aligned}
$$

are fulfilled. A pair of vector space $(\mathbf{V}, \tilde{\mathbf{V}})$ with multiplication which satisfies (1.2) and (1.11) is called a Jordan pair. This algebraic object is well known in the theory of Jordan structures (see [6]). Evidently the name "Jordan pair" has been proposed in [7] and was motivated by a close connection with Jordan algebras. One can find the detailed and systematic presentation of Jordan pairs theory in [5].

To obtain the simplest example of Jordan pair one can choose the linear space $\mathbf{M}_{p, q}(\mathbb{C})$ of $p \times q$ matrices over $\mathbb{C}$ instead of $\mathbf{V}$ and $\tilde{\mathbf{V}}$ and define the multiplication by the formula

$$
\{\mathbf{x y z}\}=\mathbf{x}^{t} \mathbf{y} \mathbf{z}+\mathbf{z}^{t} \mathbf{y} \mathbf{x},
$$

where a superscript ${ }^{t}$ means the transposition.

One can get the examples of Jordan pairs by means of the following construction (see for instance [6]) associated with the graded expansions of Lie algebras. Let $\mathbf{G}=\mathbf{G}_{1}+\mathbf{G}_{0}+\mathbf{G}_{-1}$ be the Lie algebra with $\left[\mathbf{G}_{i}, \mathbf{G}_{j}\right] \subseteq \mathbf{G}_{i+j}$. Then the pair $\left(\mathbf{G}_{1}, \mathbf{G}_{-1}\right)$ of vector spaces with multiplication

$$
\{\mathbf{x} \tilde{\mathbf{y}} \mathbf{z}\}=[[\mathbf{x}, \tilde{\mathbf{y}}], \mathbf{z}], \quad\{\tilde{\mathbf{x}} \mathbf{y} \tilde{\mathbf{z}}\}=[[\tilde{\mathbf{x}}, \mathbf{y}], \tilde{\mathbf{z}}],
$$

where $\mathbf{x}, \mathbf{y}, \mathbf{z} \in \mathbf{G}_{1}, \tilde{\mathbf{x}}, \tilde{\mathbf{y}}, \tilde{\mathbf{z}} \in \mathbf{G}_{-1}$, is a Jordan pair.

Any system (0.1) the constants $\mathbf{a}_{j k m}^{i}, \tilde{\mathbf{a}}_{j k m}^{i}$ of which satisfy the relationship (1.10) will be called a Jordan system. We shall show that all systems $(0.1)$ with nondegenerate generalized symmetries or conservation laws are exhausted by Jordan systems.

\section{The Necessary Condition for the Existence Generalized Symmetries}

In this section we will obtain the conditions on the constants $\mathbf{a}_{j k m}^{i}, \tilde{\mathbf{a}}_{j k m}^{i}$ which are necessary for the system $(0.1)$ to have the generalized symmetries. 
Let the system (0.1) have the symmetry of the order $n$

$$
\begin{aligned}
& \mathbf{u}_{\tau_{n}}^{i}=\mathbf{F}_{n}^{i}\left(\mathbf{u}, \mathbf{v}, \ldots, \mathbf{u}_{n}, \mathbf{v}_{n}\right), \\
& \mathbf{v}_{\tau_{n}}^{i}=\tilde{\mathbf{F}}_{n}^{i}\left(\mathbf{u}, \mathbf{v}, \ldots, \mathbf{u}_{n}, \mathbf{v}_{n}\right) .
\end{aligned}
$$

In accordance with the definition of the symmetry this yields the flows defined by (0.1) and (2.1) to be commuting, i.e. $\mathbf{F}_{n}^{i}, \widetilde{\mathbf{F}}_{n}^{i}$ obeys the following system of equations:

$$
\begin{aligned}
& \partial_{t}\left(\mathbf{F}_{n}^{i}\right)-\partial_{\tau_{n}}\left(\mathbf{u}_{x x}^{i}+\mathbf{a}_{j k m}^{i} \mathbf{u}^{j} \mathbf{v}^{k} \mathbf{u}^{m}\right)=, \\
& \partial_{t}\left(\tilde{\mathbf{F}}_{n}^{i}\right)+\partial_{\tau_{n}}\left(\mathbf{v}_{x x}^{i}+\tilde{\mathbf{a}}_{j k m}^{i} \mathbf{v}^{j} \mathbf{u}^{k} \mathbf{v}^{m}\right)=0 .
\end{aligned}
$$

Linearizing (2.2), i.e. taking the Freshe derivative we get the equivalent operator form of this system

$$
\left[\Lambda \mathbf{D}^{2}+\boldsymbol{\Phi}, \mathbf{L}\right]-\partial_{t}(\mathbf{L})+\partial_{\tau_{n}}(\boldsymbol{\Phi})=0
$$

Here $\Lambda$ and $\Phi$ are block matrices of the size $(N+M) \times(N+M)$

$$
\Lambda=\left(\begin{array}{cc}
\mathbf{I}_{N} & 0 \\
0 & -\mathbf{I}_{M}
\end{array}\right), \quad \boldsymbol{\Phi}=\left(\begin{array}{cc}
\mathbf{g} & \mathbf{h} \\
-\tilde{\mathbf{h}} & -\tilde{\mathbf{g}}
\end{array}\right),
$$

$\mathbf{I}_{N}$ and $\mathbf{I}_{M}$ are unit matrices of the sizes $N \times N$ and $M \times M$, respectively, $\mathbf{g}, \tilde{\mathbf{g}}$, and $\mathbf{h}$ matrices of the sizes $N \times N, M \times M, N \times M, M \times N$ with the elements defined by right-hand side of the system (0.1) according to the formulae

$$
\begin{array}{ll}
(\mathbf{g})_{m}^{i}=2 \mathbf{a}_{j k m}^{i} \mathbf{u}^{j} \mathbf{v}^{k}, & (\tilde{\mathbf{g}})_{m}^{i}=2 \tilde{\mathbf{a}}_{j k m}^{i} \mathbf{v}^{j} \mathbf{u}^{k}, \\
(\mathbf{h})_{m}^{i}=\mathbf{a}_{j m k}^{i} \mathbf{u}^{j} \mathbf{u}^{k}, & (\tilde{\mathbf{h}})_{m}^{i}=\tilde{\mathbf{a}}_{j m k}^{i} \mathbf{v}^{j} \mathbf{v}^{k} .
\end{array}
$$

Here $\mathbf{L}$ is the differential operator

$$
\mathbf{L}=\sum_{i=0}^{n} \mathbf{L}_{i} \mathbf{D}^{i}
$$

with the matrix coefficients $\mathbf{L}_{i}$ which are written in block form

$$
\mathbf{L}_{i}=\left(\begin{array}{cc}
\mathbf{l}_{i} & \mathbf{s}_{i} \\
\tilde{\mathbf{s}}_{i} & \tilde{\mathbf{I}}_{i}
\end{array}\right)
$$

where $\mathbf{l}_{i}, \mathfrak{I}_{i}, \mathbf{s}_{i}, \tilde{\mathbf{s}}_{i}$ are matrices of the sizes $N \times N, M \times M, N \times M, M \times N$. Their elements are defined by the right-hand side of the symmetry (2.1) in accordance with the following formulae:

$$
\begin{array}{rlrl}
\left(\mathbf{l}_{i}\right)_{m}^{k} & =\partial \mathbf{F}^{k} / \partial \mathbf{u}_{i}^{m}, & \left(\mathbf{s}_{i}\right)_{m}^{k}=\partial \mathbf{F}^{k} / \partial \mathbf{v}_{i}^{m}, \\
\left(\tilde{\mathbf{s}}_{i}\right)_{m}^{k}=\partial \tilde{\mathbf{F}}^{k} / \partial \mathbf{u}_{i}^{m}, & \left(\widetilde{I}_{i}\right)_{m}^{k}=\partial \widetilde{\mathbf{F}}^{k} / \partial \mathbf{v}_{i}^{m} .
\end{array}
$$

Differentiation by $t$ is made according to (0.1) while the differentiation by $\tau_{n}-$ according to (2.1),

$$
\partial_{t}(\mathbf{L}) \stackrel{\text { def }}{=} \sum_{i=0}^{n} \partial_{t}\left(\mathbf{L}_{i}\right) \mathbf{D}^{i}
$$

It's easy to check that

$$
\left[\Lambda \mathbf{D}^{2}+\boldsymbol{\Phi}, \mathbf{L}\right]-\partial_{t}(\mathbf{L})+\partial_{\tau_{n}}(\mathbf{\Phi})=\sum_{i=0}^{n+2} \dot{\mathbf{M}}_{i} \mathbf{D}^{i}
$$


where $\mathbf{M}_{i}$ are of the form

$$
\begin{gathered}
\mathbf{M}_{n+2}=\left[\Lambda, \mathbf{L}_{n}\right], \quad \mathbf{M}_{n+1}=2 \Lambda \mathbf{D}\left(\mathbf{L}_{n}\right)+\left[\Lambda, \mathbf{L}_{n-1}\right], \\
\mathbf{M}_{n}=\Lambda \mathbf{D}^{2}\left(\mathbf{L}_{n}\right)+2 \Lambda \mathbf{D}\left(\mathbf{L}_{n-1}\right)+\left[\Lambda, \mathbf{L}_{n-2}\right]+\left[\boldsymbol{\Phi}, \mathbf{L}_{n}\right]-\partial_{t}\left(\mathbf{L}_{n}\right)
\end{gathered}
$$

and so on. Operator equation (2.3) is equivalent to the system of matrix equations $\mathbf{M}_{i}=0(i=n+2, n+1, \ldots, 1,0)$ with respect to the undefined coefficients of the differential operator $\mathbf{L}$. Let us show that matrices $\mathbf{L}_{i}$ may be successively defined and let us explain how the conditions for $\mathbf{a}_{j k m}^{i}$ and $\tilde{\mathbf{a}}_{j k m}^{i}$ arise.

During the calculation it is convenient to use the block structure of matrices $\Lambda, \mathbf{L}_{i}, \boldsymbol{\Phi}$. From the equation $\mathbf{M}_{n+2}=0$ we get

$$
\mathbf{S}_{n}=0, \quad \tilde{\mathbf{s}}_{n}=0 .
$$

The equation $\mathbf{M}_{n+1}=0$ is equivalent to

$$
\mathbf{s}_{n-1}=0, \quad \tilde{\mathbf{s}}_{n-1}=0, \quad \mathbf{D}\left(\mathbf{l}_{n}\right)=0, \quad \mathbf{D}\left(\tilde{\mathbb{I}}_{n}\right)=0 .
$$

Therefore, $\mathbf{I}_{n}$ and $\mathbb{I}_{n}$ are the constant matrices. Let's denote $\mathbf{I}_{n}=\mathbf{K}, \mathfrak{I}_{n}=\tilde{\mathbf{K}}$. It's easy to see that the matrices $\mathbf{L}_{i}$ may be recursively defined, namely each equation $\mathbf{M}_{i}=0$ with $i \geqq 2$ is equivalent to

$$
\begin{array}{cl}
\mathbf{s}_{i-2}=\kappa_{i-2}, & \tilde{\mathbf{s}}_{i-2}=\tilde{\kappa}_{i-2}, \\
\mathbf{D}\left(\mathbf{l}_{i-1}\right)=\eta_{i-1}, & \mathbf{D}\left(\tilde{\mathbf{L}}_{i-1}\right)=\tilde{\eta}_{i-1},
\end{array}
$$

where $\kappa_{i-2}, \tilde{\kappa}_{i-2}, \eta_{i-1}, \tilde{\eta}_{i-1}$ are already known matrices with the elements depending on $\mathbf{u}^{i}, \mathbf{v}^{i}$ and on their derivatives by $x$. It's clear that the equations of the form $\mathbf{D}(\mathbf{X})=\eta$ are solvable not for any right-hand side $\eta$. Therefore, while solving Eqs. (2.10) we, generally speaking, will obtain the conditions for the constant of the system $(0.1)$. The constants of integrations arising from the solving of the previous equations have the same form.

Proposition 2.1. Each symmetry of the order $n$ for (0.1) has the form

$$
\begin{aligned}
& \mathbf{u}_{\tau_{n}}^{i}=\mathbf{K}_{j}^{i} \mathbf{u}_{n}^{j}+\mathbf{f}_{n}^{i}\left(\mathbf{u}, \mathbf{v}, \ldots, \mathbf{u}_{n-1}, \mathbf{v}_{n-1}\right), \\
& \mathbf{v}_{\tau_{n}}^{i}=\widetilde{\mathbf{K}}_{j}^{i} \mathbf{v}_{n}^{j}+\widetilde{\mathbf{f}}_{n}^{i}\left(\mathbf{u}, \mathbf{v}, \ldots, \mathbf{u}_{n-1}, \mathbf{v}_{n-1}\right),
\end{aligned}
$$

where $\mathbf{K}_{j}^{i}, \widetilde{\mathbf{K}}_{j}^{i}$ are constants while functions $\mathbf{f}_{n}^{i}$ and $\mathbf{f}_{n}^{i}$ are polynomials of all their arguments.

Proof. The right-hand side of the symmetry may be reconstructed by the coefficients of $\mathbf{L}$ with the help of (2.5). It's clear that if elements of all matrices $\mathbf{L}_{i}$ are polynomials then the right-hand side of the symmetry polynomially depends on all of its arguments. Matrices $\kappa_{i-2}, \tilde{\kappa}_{i-2}, \eta_{i-1}, \tilde{\eta}_{i-1}$ in (2.9) and (2.10) are polynomials as they are obtained by differentiation and multiplication of polynomial matrices. Therefore, all matrices $\mathbf{L}_{i}$ are polynomials of their arguments. The matter that the symmetry has the form (2.11) is the consequence of formulae (2.7) and (2.8).

Let's call the symmetry nondegenerate if $\mathbf{K}$ and $\widetilde{\mathbf{K}}$ are nondegenerate matrices.

The system (0.1) is homogeneous, i.e. it is invariant with respect to the oneparameter scaling group $\Omega_{\lambda}$,

$$
\tilde{x}=\lambda x, \quad \tilde{t}=\lambda^{2} t, \quad \tilde{\mathbf{u}}^{i}=\lambda \mathbf{u}^{i}, \quad \tilde{\mathbf{v}}^{i}=\lambda \mathbf{v}^{i} .
$$


Let's call the polynomial $\mathbf{P}\left(\mathbf{u}, \mathbf{v}, \ldots, \mathbf{u}_{k}, \mathbf{v}_{k}\right)$ to be homogeneous if $\Omega_{\lambda}\left(\mathbf{P}\left(\mathbf{u}, \mathbf{v}, \ldots, \mathbf{u}_{k}, \mathbf{v}_{k}\right)\right)$ $=\lambda^{\gamma} \mathbf{P}\left(\mathbf{u}, \mathbf{v}, \ldots, \mathbf{u}_{k}, \mathbf{v}_{k}\right)$. Here $\gamma$ is called the weight of $\mathbf{P}$ and denoted as $\gamma=\omega(\mathbf{P})$. The concept of homogenity may be generalized up to the case of differential operators by letting $\omega(\mathbf{D})=1$. It's easy to check that

$$
\begin{aligned}
\omega\left(\mathbf{u}_{k}^{i}\right) & =k+1, \quad \omega\left(\mathbf{v}_{k}^{i}\right)=k+1, \quad \omega(\mathbf{P Q})=\omega(\mathbf{P})+\omega(\mathbf{Q}), \\
\omega(\mathbf{D}(\mathbf{P})) & =\omega(\mathbf{P})+1, \quad \omega\left(\partial_{t}(\mathbf{P})\right)=\omega(\mathbf{P})+2, \\
\omega\left(\mathbf{P D}^{k}\right) & =\omega(\mathbf{P})+k
\end{aligned}
$$

for any homogeneous polynomials $\mathbf{P}$ and $\mathbf{Q}$. Each non-homogeneous polynomial is the sum of its homogeneous components, i.e. the polynomials with the fixed weights.

Suppose the system (0.1) has the symmetry (2.1) with the polynomial right-hand side. Let's consider the system $\mathbf{u}_{\tau}^{i}=\mathbf{H}^{i}, \mathbf{v}_{\tau}^{i}=\tilde{\mathbf{H}}^{i}$, where $\mathbf{H}^{i}, \tilde{\mathbf{H}}^{i}$ are the homogeneous components of polynomials $\mathbf{F}^{i}$ and $\tilde{\mathbf{F}}^{i}$, respectively, with the same weights. With the help of (2.13) one can find that such a system is also the symmetry. Symmetry with homogeneous right-hand side we will call the homogeneous symmetry. Obviously the homogeneous symmetry (2.11) is invariant with respect to the one parameter scaling group $\Omega_{\lambda}(n)$

$$
\tilde{x}=\lambda x, \quad \tau=\lambda^{n} \tau, \quad \tilde{\mathbf{u}}^{i}=\lambda \mathbf{u}^{i}, \quad \tilde{\mathbf{v}}^{i}=\lambda \mathbf{v}^{i},
$$

while polynomials $\mathbf{f}_{n}, \mathfrak{f}_{n}$ are of weight $n+1$. In what follows we can consider only the homogeneous symmetries without loss of generality. In this case the matrix elements of $\mathbf{L}_{i}$ are homogeneous polynomials with the weight $n-i$. Generally speaking matrices $\mathbf{I}_{i}, \mathbf{I}_{i}$ with $i<n$ are derived from (2.10) up to the additive "constants of integration" being the constant matrices. Considering the homogeneous symmetry one should take them to be zero for the sake of simplicity.

Proposition 2.2. For the system (0.1) to have the symmetry (2.11) of the order $n \geqq 1$ it's necessary and sufficient for the equalities

$$
\begin{array}{rlrl}
\mathbf{K}_{r}^{i} \mathbf{a}_{j k m}^{r}-\mathbf{a}_{j k r}^{i} \mathbf{K}_{m}^{r} & =0, & \tilde{\mathbf{K}}_{r}^{i} \tilde{\mathbf{a}}_{j k m}^{r}-\tilde{\mathbf{a}}_{j k k}^{i} \tilde{\mathbf{K}}_{m}^{r}=0, \\
\mathbf{K}_{r}^{i} \mathbf{a}_{j k m}^{r}+(-1)^{n} \mathbf{a}_{j r m}^{i} \tilde{\mathbf{K}}_{k}^{r}=0, & \tilde{\mathbf{K}}_{r}^{i} \tilde{\mathbf{a}}_{j k m}^{r}+(-1)^{n} \tilde{\mathbf{a}}_{j r m}^{i} \mathbf{K}_{k}^{r}=0 .
\end{array}
$$

to be held for any indices $i, j, k, m$.

Proof. From the matrix equation $\mathbf{M}_{n}=0$ we get

$$
\mathbf{s}_{n-2}=1 / 2(\mathbf{K h}-\mathbf{h} \tilde{\mathbf{K}}), \quad \tilde{\mathbf{s}}_{n-2}=1 / 2(\tilde{\mathbf{K}} \tilde{\mathbf{h}}-\tilde{\mathbf{h}} \mathbf{K}), \quad[\mathbf{K}, \mathbf{g}]=0, \quad[\tilde{\mathbf{K}}, \tilde{\mathbf{g}}]=0 .
$$

The last two equalities are equivalent to (2.14). It's not too easy to get (2.15). Let's introduce the matrices $\mathbf{A}(j, k), \tilde{\mathbf{A}}(j, k)$, which are defined by

$$
(\mathbf{A}(j, k))_{m}^{i}=\mathbf{a}_{j m k}^{i}, \quad(\tilde{\mathbf{A}}(j, k))_{m}^{i}=\tilde{\mathbf{a}}_{j m k}^{i} .
$$

Moreover, let's denote $\mathbf{B}(j, k) \stackrel{\text { def }}{=} \mathbf{K A}(j, k))-\mathbf{A}(j, k) \tilde{\mathbf{K}}$. In terms of such definitions $\mathbf{s}_{n-2}=1 / 2 \mathbf{B}(j, k) \mathbf{u}^{j} \mathbf{u}^{k}$. Matrices $\mathbf{s}_{i}$ which are recursively defined by equations $\mathbf{M}_{i}=0$ are the homogeneous polynomials. Let's denote via $\mathbf{s}_{i}^{*}$ the quadratic part of these matrices. From $\mathbf{M}_{i}=0$ one can get the recurrent formula for $\mathbf{s}_{i}^{*}$ with $i=n-3, \ldots, 0$ :

$$
\mathbf{s}_{i}^{*}=1 / 2\left(\mathbf{C}_{n}^{i+2} \mathbf{D}^{n-i-2}(\mathbf{g})+\partial_{t}\left(\mathbf{s}_{i+2}^{*}\right)-2 \mathbf{D}\left(\mathbf{s}_{i+1}^{*}\right)-\mathbf{D}^{2}\left(\mathbf{s}_{i+2}^{*}\right)\right) .
$$


It is easy to check using induction that this formula leads to

$$
\begin{aligned}
\mathbf{s}_{n-2 i-2}^{*}= & 1 / 2(-1)^{i} \mathbf{B}(j, k) \mathbf{u}_{i}^{j} \mathbf{u}_{i}^{k}+\mathbf{D}^{2}\left(\varphi_{n-2 i-2}\right), \\
\mathbf{s}_{n-2 i-1}^{*}= & 1 / 2(-1)^{i} \mathbf{D}\left((i \mathbf{B}(j, k)-n \mathbf{K} \mathbf{A}(j, k)) \mathbf{u}_{i-1}^{j} \mathbf{u}_{i-1}^{k}\right) \\
& +\mathbf{D}^{2}\left(\varphi_{n-2 i-1}\right) .
\end{aligned}
$$

We will not interest in the explicit form of matrices $\varphi_{j}$ depending on $\mathbf{u}^{i}$ and on their derivatives by $x$. From $\mathbf{M}_{1}=0$ we obtain that $\mathbf{s}_{1}^{*}$ and $\mathbf{s}_{0}^{*}$ should obey the following condition:

$$
n \mathbf{D}^{n-1}(\mathbf{g})+\partial_{t}\left(\mathbf{s}_{1}^{*}\right)-2 \mathbf{D}\left(s_{0}^{*}\right)-\mathbf{D}^{2}\left(\mathbf{s}_{1}^{*}\right)=0 .
$$

Using (2.17) we get that if $n=2 m$ then Eq. (2.18) can be rewritten in the form

$$
\mathbf{D}\left((2 m \mathbf{K} \mathbf{A}(j, k)-m \mathbf{B}(j, k)) \mathbf{u}_{m-1}^{j} \mathbf{u}_{m-1}^{k}\right)+\mathbf{D}^{2}(\varphi)=0 .
$$

This yields $2 \mathbf{K A}(j, k)-\mathbf{B}(j, k)=0$ which in its turn is equivalent to the first condition (2.15). If $n=2 m+1$ then Eq. (2.18) is written in the form

$$
\mathbf{B}(j, k) \mathbf{u}_{m}^{j} \mathbf{u}_{m}^{k}+\mathbf{D}^{2}(\varphi)=0,
$$

From here we get $\mathbf{B}(j, k)=0$ which is just so the other form of the first condition (2.15). The necessity of the second condition (2.15) may be proved in the same way.

Remark 2.1. One can check directly that the conditions (2.14) and (2.15) are enough for the existence of the homogeneous symmetry of the first order having the form

$$
\mathbf{u}_{\tau_{1}}^{i}=\mathbf{K}_{j}^{i} \mathbf{u}_{x}^{j}, \quad \mathbf{v}_{\tau_{1}}^{i}=\widetilde{\mathbf{K}}_{j}^{i} \mathbf{v}_{x}^{j} .
$$

Remark 2.2. It's easy to see that conditions (2.14) and (2.15) hold for arbitrary values of constants $\mathbf{a}_{j k m}^{i}, \tilde{\mathbf{a}}_{j k m}^{i}$ if one takes $\mathbf{K}=\mathbf{I}_{N}, \widetilde{\mathbf{K}}=\mathbf{I}_{M}$ for the symmetries of odd order and $\mathbf{K}=\mathbf{I}_{N}, \widetilde{\mathbf{K}}=-\mathbf{I}_{M}$ for the symmetries of even order.

Let's give the algebraic interpretation for the conditions obtained. Let $\mathbf{K}: \mathbf{V} \rightarrow \mathbf{V}$ and $\tilde{\mathbf{K}}: \tilde{\mathbf{V}} \rightarrow \tilde{\mathbf{V}}$ be the linear operators defined by $\mathbf{K} \mathbf{e}_{j}=\mathbf{K}_{j}^{i} \mathbf{e}_{i}, \widetilde{\mathbf{K}} \tilde{\mathbf{e}}_{j}=\widetilde{\mathbf{K}}_{j}^{i} \tilde{\mathbf{e}}_{i}$. Then Eqs. (2.14) and (2.15) mean that for all $\mathbf{x}, \mathbf{y}, \mathbf{z} \in \mathbf{V}, \tilde{\mathbf{x}}, \tilde{\mathbf{y}}, \tilde{\mathbf{z}} \in \tilde{\mathbf{V}}$ the equations

$$
\begin{aligned}
\mathbf{K}\{\mathbf{x} \tilde{\mathbf{y}} \mathbf{z}\}=\{\mathbf{x} \tilde{\mathbf{y}} \mathbf{K z}\}, & \tilde{\mathbf{K}}\{\tilde{\mathbf{x}} \mathbf{y} \tilde{\mathbf{z}}\}=\{\tilde{\mathbf{x}} \mathbf{y} \tilde{\mathbf{K}} \mathbf{z}\}, \\
\mathbf{K}\{\mathbf{x} \tilde{\mathbf{y} z}\}=(-1)^{n+1}\{\mathbf{x} \tilde{\mathbf{K}} \tilde{\mathbf{y} z}\}, & \widetilde{\mathbf{K}}\{\tilde{\mathbf{x}} \mathbf{y} \tilde{\mathbf{z}}\}=(-1)^{n+1}\{\tilde{\mathbf{x}} \mathbf{K} \mathbf{y} \tilde{\mathbf{z}}\}
\end{aligned}
$$

hold.

Proposition 2.3. Each system (0.1) possessing the degenerate symmetry of the order $n \geqq 1$ is reducible.

Proof. Let the matrix $\mathbf{K}$ be degenerate and let $\mathbf{V}_{0} \in \mathbf{V}$ be the eigenspace corresponding to the zero eigenvalue. Then from (2.20) and (2.21) we get $\left(\mathbf{V}_{0}, \tilde{\mathbf{V}}\right)$ to be the ideal, and hence according to Proposition 1.1 the corresponding system $(0.1)$ is reducible.

The conditions obtained though aren't enough for the system $(0.1)$ to have the generalized symmetries. Defining recursively the coefficients of the differential 
operator $\mathbf{L}$ we find that for the solvability of the equations $\mathbf{M}_{n-2}=0, \mathbf{M}_{n-3}=0$ it is necessary to demand the existence of the solution $\sigma_{1}\left(\mathbf{u}, \mathbf{v}, \mathbf{u}_{x}, \mathbf{v}_{x}\right), \tilde{\sigma}_{1}\left(\mathbf{u}, \mathbf{v}, \mathbf{u}_{x}, \mathbf{v}_{x}\right)$, $\sigma_{2}\left(\mathbf{u}, \mathbf{v}, \mathbf{u}_{x}, \mathbf{v}_{x}, \mathbf{u}_{x x}, \mathbf{v}_{x x}\right), \tilde{\sigma}_{2}\left(\mathbf{u}, \mathbf{v}, \mathbf{u}_{x}, \mathbf{v}_{x}, \mathbf{u}_{x x}, \mathbf{v}_{x x}\right)$ of the system of matrix equations

$$
\begin{aligned}
\mathbf{K}\left(\partial_{t}(\mathbf{g})-\mathbf{D}\left(\sigma_{1}\right)\right) & =0, & & \widetilde{\mathbf{K}}\left(\partial_{t}(\tilde{\mathbf{g}})-\mathbf{D}\left(\tilde{\sigma}_{1}\right)\right)=0, \\
\mathbf{K}\left(\partial_{t}\left(\sigma_{1}\right)-\left[\mathbf{g}, \sigma_{1}\right]-\mathbf{D}\left(\sigma_{2}\right)\right) & =0, & & \widetilde{\mathbf{K}}\left(\partial_{t}\left(\tilde{\sigma}_{1}\right)+\left[\tilde{\mathbf{g}}, \tilde{\sigma}_{1}\right]-\mathbf{D}\left(\tilde{\sigma}_{2}\right)\right)=0 .
\end{aligned}
$$

Note that the conditions (2.22) mean that each element of matrices $\mathbf{K g}$ and $\tilde{\mathbf{K}} \tilde{\mathbf{g}}$ should be the conservation density for the system (0.1).

Proposition 2.4. Equations (2.22) and (2.23) are solvable if and only if the following equations

$$
\begin{gathered}
\mathbf{K}_{r}^{i}\left(\mathbf{a}_{j k n}^{r} \mathbf{a}_{m s p}^{n}-\mathbf{a}_{m s n}^{r} \mathbf{a}_{j k p}^{n}-\mathbf{a}_{n s p}^{r} \mathbf{a}_{j k m}^{n}+\mathbf{a}_{m n}^{r} \tilde{\mathbf{a}}_{k j s}^{n}\right)=0, \\
\tilde{\mathbf{K}}_{r}^{i}\left(\tilde{\mathbf{a}}_{j k n}^{r} \tilde{\mathbf{a}}_{m s p}^{n}-\tilde{\mathbf{a}}_{m s n}^{r} \tilde{\mathbf{a}}_{j k p}^{n}-\tilde{\mathbf{a}}_{n s p}^{r} \tilde{\mathbf{a}}_{j k m}^{n}+\tilde{\mathbf{a}}_{m n p}^{r} \mathbf{a}_{k j s}^{n}\right)=0
\end{gathered}
$$

hold for all $i, j, k, m, s, p$.

Proof. For the sake of brevity it's convenient to introduce the matrices $\mathbf{a}(j, k), \tilde{\mathbf{a}}(j, k)$ via the formulae

$$
(\mathbf{a}(j, k))_{m}^{i}=\mathbf{a}_{j k m}^{i}, \quad(\tilde{\mathbf{a}}(j, k))_{m}^{i}=\tilde{\mathbf{a}}_{j k m}^{i} .
$$

Then $\mathbf{g}=2 \mathbf{a}(j, k) \mathbf{u}^{j} \mathbf{v}^{k}, \quad \tilde{\mathbf{g}}=2 \tilde{\mathbf{a}}(j, k) \mathbf{v}^{j} \mathbf{u}^{k}$.

From the first Eq. (2.22) we get

$$
\mathbf{K} \sigma_{1}=2 \mathbf{K a}(j, k)\left(\mathbf{v}^{k} \mathbf{u}_{x}^{j}-\mathbf{u}^{j} \mathbf{v}_{x}^{k}\right)+\mathbf{K} \kappa,
$$

where $\kappa$ is the solution of the following matrix equation:

$$
\mathbf{K D}(\kappa)=2 \mathbf{K}\left(\mathbf{a}_{j k m}^{n} \mathbf{a}(n, s)-\tilde{\mathbf{a}}_{k j \mathbf{s}}^{n} \mathbf{a}(m, n)\right) \mathbf{u}^{j} \mathbf{u}^{m} \mathbf{v}^{k} \mathbf{v}^{s} .
$$

This equation is solvable if and only if its right-hand side is zero, i.e. constants satisfy the following constraint:

$$
\mathbf{K}\left(\mathbf{a}_{j k m}^{n} \mathbf{a}(n, s)+\mathbf{a}_{j s m}^{n} \mathbf{a}(n, k)-\tilde{\mathbf{a}}_{k j s}^{n} \mathbf{a}(m, n)-\tilde{\mathbf{a}}_{k m s}^{n} \mathbf{a}(j, n)\right)=0,
$$

as we are considering the homogeneous symmetry $\kappa=0$.

From the first Eq. (2.23) we get

$$
\mathbf{K} \sigma_{2}=2 \mathbf{K a}(j, k)\left(\mathbf{v}^{k} \mathbf{u}_{x x}^{j}+\mathbf{u}^{j} \mathbf{v}_{x x}^{k}-2 \mathbf{v}_{x}^{k} \mathbf{u}_{x}^{j}\right)+\mathbf{K} \kappa,
$$

where the matrix $\kappa$ satisfies the equation $\mathbf{K D}(\kappa)=\mathbf{H}$ with

$$
\begin{aligned}
\mathbf{H}= & 2 \mathbf{K}\left(2 \mathbf{a}_{j k m}^{n} \mathbf{a}(n, s)+\tilde{\mathbf{a}}_{k m s}^{n} \mathbf{a}(j, n)-\tilde{\mathbf{a}}_{k j s}^{n} \mathbf{a}(m, n)\right. \\
& +[\mathbf{a}(m, k), \mathbf{a}(j, s)]) \mathbf{v}^{s} \mathbf{v}^{s} \mathbf{u}^{j} \mathbf{u}_{x}^{m}+2 \mathbf{K}\left(2 \tilde{\mathbf{a}}_{k j s}^{n} \mathbf{a}(m, n)\right. \\
& \left.+\mathbf{a}_{j s m}^{n} \mathbf{a}(n, k)-\mathbf{a}_{j k m}^{n} \mathbf{a}(n, s)+[\mathbf{a}(m, k), \mathbf{a}(j, s)]\right) \mathbf{u}^{j} \mathbf{u}^{m} \mathbf{v}^{k} \mathbf{v}_{x}^{s} .
\end{aligned}
$$

This equation is solvable if and only if for all indices $j, k$ the following conditions hold:

$$
\begin{gathered}
\partial^{2} \mathbf{H} / \partial \mathbf{u}^{j} \partial \mathbf{u}_{x}^{k}=\partial^{2} \mathbf{H} / \partial \mathbf{u}^{k} \partial \mathbf{u}_{x}^{j}, \quad \partial^{2} \mathbf{H} / \partial \mathbf{v}^{j} \partial \mathbf{v}_{x}^{k}=\partial^{2} \mathbf{H} / \partial \mathbf{v}^{k} \partial \mathbf{v}_{x}^{j}, \\
\partial^{2} \mathbf{H} / \partial \mathbf{u}^{j} \partial \mathbf{v}_{x}^{k}=\partial^{2} \mathbf{H} / \partial \mathbf{v}^{k} \partial \mathbf{u}_{x}^{j} .
\end{gathered}
$$


One can check that they are equivalent to the constraints

$$
\begin{gathered}
\mathbf{K}[\mathbf{a}(j, k), \mathbf{a}(m, s)]+\mathbf{K}[\mathbf{a}(j, s), \mathbf{a}(m, k)] \\
=\tilde{\mathbf{a}}_{k m s}^{n} \mathbf{K a}(j, n)-\tilde{\mathbf{a}}_{k j s}^{n} \mathbf{K a}(m, n), \\
\mathbf{K}[\mathbf{a}(j, k), \mathbf{a}(m, s)]-\mathbf{K}[\mathbf{a}(j, s), \mathbf{a}(m, k)] \\
=\mathbf{a}_{j k m}^{n} \mathbf{K a}(n, s)-\mathbf{a}_{j s m}^{n} \mathbf{K a}(n, k), \\
\mathbf{K}[\mathbf{a}(j, k), \mathbf{a}(m, s)]=\mathbf{a}_{j k m}^{n} \mathbf{K a}(n, s)-\tilde{\mathbf{a}}_{k j s}^{n} \mathbf{K a}(m, n)
\end{gathered}
$$

for the constants. Writing the matrix equation (2.30) in its components we get (2.24). Equations (2.27)-(2.29) are its consequences. This follows from the matrix form of (2.30).

Necessity and sufficiency of $(2.25)$ is proved in the same way.

Remark 2.3. Equations (2.24) and (2.25) were obtained supposing the system (0.1) to have a symmetry of the order $n \geqq 4$. One may check by direct calculations that conditions (2.14), (2.15), (2.24), (2.25) are necessary and sufficient for the system (0.1) to have the symmetry of the order $n=3$. The homogeneous symmetry of the third order has the form

$$
\begin{aligned}
& \mathbf{u}_{\tau_{3}}^{i}=\mathbf{K}_{r}^{i} \mathbf{u}_{x x x}^{r}+3 \mathbf{K}_{r}^{i} \mathbf{a}_{j k m}^{r} \mathbf{u}^{j} \mathbf{v}^{k} \mathbf{u}_{x}^{m}, \\
& \mathbf{v}_{\tau_{3}}^{i}=\widetilde{\mathbf{K}}_{r}^{i} \mathbf{v}_{x x x}^{r}+3 \widetilde{\mathbf{K}}_{r}^{i} \tilde{\mathbf{a}}_{j k m}^{r} \mathbf{v}^{j} \mathbf{u}^{k} \mathbf{v}_{x}^{m}
\end{aligned}
$$

[compare with (1.9)].

In terms of vector spaces with triple multiplication the constraints (24) and (25) may be rewritten in the form

$$
\begin{aligned}
& \mathbf{K}(\{\mathbf{x} \tilde{\mathbf{y}}\{\mathbf{y} \tilde{\mathbf{x}} \mathbf{z}\}\}-\{\mathbf{y} \tilde{\mathbf{x}}\{\mathbf{x} \tilde{\mathbf{y}} \mathbf{z}\}\}-\{\mathbf{z} \tilde{\mathbf{x}}\{\mathbf{x} \tilde{\mathbf{y}} \mathbf{y}\}\}+\{\mathbf{y}\{\tilde{\mathbf{y}} \mathbf{x} \hat{\mathbf{x}}\} \mathbf{z}\})=0, \\
& \tilde{\mathbf{K}}(\{\tilde{\mathbf{x}} \mathbf{y}\{\tilde{\mathbf{y}} \mathbf{x} \tilde{\mathbf{z}}\}\}-\{\tilde{\mathbf{y}} \mathbf{x}\{\tilde{\mathbf{x}} \mathbf{y} \tilde{\mathbf{z}}\}\}-\{\tilde{\mathbf{z}} \mathbf{x}\{\tilde{\mathbf{x}} \mathbf{y} \tilde{\mathbf{y}}\}\}+\{\tilde{\mathbf{y}}\{\mathbf{y} \tilde{\mathbf{x}} \mathbf{x}\} \tilde{\mathbf{z}}\})=0 .
\end{aligned}
$$

For nondegenerate operators $\mathbf{K}$ and $\widetilde{\mathbf{K}}$ (1.11) follows from (2.32), i.e. the appropriate system is the Jordan system. In the opposite case from (2.20) and (2.21) we find the pair $(\operatorname{Ker} \mathbf{K}, \operatorname{Ker} \widetilde{\mathbf{K}})$ to be the ideal in the pair $(\mathbf{V}, \widetilde{\mathbf{V}})$. Then from (2.32) we obtain that the factor pair $(\mathbf{V}, \tilde{\mathbf{V}}) /(\operatorname{Ker} \mathbf{K}, \operatorname{Ker} \widetilde{\mathbf{K}})$ is the Jordan system. Thus we proved the main statement of this chapter.

Theorem 2.1. Systems (0.1) with the nondegenerate generalized symmetry are exhausted by Jordan systems. System (0.1) with the degenerate symmetry is reducible, the factor pair $(\mathbf{V}, \widetilde{\mathbf{V}}) /(\operatorname{Ker} \mathbf{K}, \operatorname{Ker} \widetilde{\mathbf{K}})$ being the Jordan system.

\section{The Necessary Condition for the Existence Conservation Laws}

Let's recall that the local conservation law of the system $(0.1)$ is the equation

$$
\partial_{t}(\varrho)=\mathbf{D}(\sigma) \text {, }
$$

where $\varrho, \sigma$ are functions of $\mathbf{u}^{i}, \mathbf{v}^{i}$ and the finite number of their derivatives by $x$. The function $\varrho$ is usually called the density, $\sigma$ is called the flux. Let's investigate the question of what the constants should be to make the system (0.1) have the conservation laws. As in the case of symmetries we find it convenient to transfer by 
the usual way from (3.1) to the equivalent operator equation. Let's define the variational derivatives $\delta \varrho / \delta \mathbf{u}^{i}, \delta \varrho / \delta \mathbf{v}^{i}$ in the following way:

$$
\delta \varrho / \delta \mathbf{u}^{i} \stackrel{\text { def }}{=} \sum_{k}(-1)^{k} \mathbf{D}^{k}\left(\partial \varrho / \partial \mathbf{u}_{k}^{i}\right), \quad \delta \varrho / \delta \mathbf{v}^{i} \stackrel{\text { def }}{=} \sum_{k}(-1)^{k} \mathbf{D}^{k}\left(\partial \varrho / \partial \mathbf{v}_{k}^{i}\right) .
$$

Then the vector-function

$$
\mathbf{P}=\left(\delta \varrho / \delta \mathbf{u}^{1}, \ldots, \delta \varrho / \delta \mathbf{u}^{N}, \delta \varrho / \delta \mathbf{v}^{1}, \ldots, \delta \varrho / \delta \mathbf{v}^{M}\right)^{\tau}
$$

obey the equation

$$
\partial_{t}(\mathbf{P})+\left(\Lambda \mathbf{D}^{2}+\boldsymbol{\Phi}^{\tau}\right)(\mathbf{P})=0
$$

where $\boldsymbol{\Phi}^{\tau}$ is a transposition of $\boldsymbol{\Phi}$ while $\Lambda$ and $\boldsymbol{\Phi}$ are defined by (2.4). Linearizing (3.2) we get the operator equation

$$
\partial_{t}(\mathbf{R})+\mathbf{R}\left(\Lambda \mathbf{D}^{2}+\boldsymbol{\Phi}\right)+\left(\Lambda \mathbf{D}^{2}+\boldsymbol{\Phi}^{\tau}\right) \mathbf{R}-\mathbf{S}=0 .
$$

Let's explain the notations: $\mathbf{R}$ is a differential operator -

$$
\mathbf{R}=\sum_{i=0}^{m} \mathbf{R}_{i} \mathbf{D}^{i}
$$

with the matrix coefficients written in the block form

$$
\mathbf{R}_{i}=\left(\begin{array}{cc}
\mathbf{r}_{i} & \mathbf{p}_{i} \\
\tilde{\mathbf{p}}_{i} & \tilde{\mathbf{r}}_{i}
\end{array}\right)
$$

with the matrices $\mathbf{r}_{i}, \tilde{\mathbf{r}}_{i}, \mathbf{p}_{i}, \tilde{\mathbf{p}}_{i}$ being of the sizes $N \times N, M \times M, N \times M, M \times N$. Their elements are defined by the density of the conservation law $\varrho$ as follows:

$$
\begin{aligned}
& \left(\mathbf{r}_{i}\right)_{m}^{k}=\partial\left(\delta \varrho / \delta \mathbf{u}^{k}\right) / \partial \mathbf{u}_{i}^{m}, \quad\left(\mathbf{p}_{i}\right)_{m}^{k}=\partial\left(\delta \varrho / \delta \mathbf{u}^{k}\right) / \partial \mathbf{v}_{i}^{m}, \\
& \left(\tilde{\mathbf{p}}_{i}\right)_{m}^{k}=\partial\left(\delta \varrho / \delta \mathbf{v}^{k}\right) / \partial \mathbf{u}_{i}^{m},\left(\tilde{\mathbf{r}}_{i}\right)_{m}^{k}=\partial\left(\delta \varrho / \delta \mathbf{v}^{k}\right) / \partial \mathbf{v}_{i}^{m} .
\end{aligned}
$$

Here $\mathbf{S}$ is the differential operator of zero degree, i.e. simply the matrix the explicit form of which doesn't matter in what follows.

The degree of the differential operator $\mathbf{R}$ with the coefficients defined by (3.4) is called the degree of the conservation law with the density @. Note that $\mathbf{R}$ satisfy the relationship

$$
\mathbf{R}^{T}-(-1)^{m} \mathbf{R}=0,
$$

as a consequence of its definition. Here $\mathbf{R}^{T}$ is a formally adjoint operator

$$
\mathbf{R}^{T}=\sum_{i=0}^{m}(-1)^{i} \mathbf{D}^{i} \mathbf{R}_{i}^{\tau}
$$

Let's call the conservation law with the density $\varrho$ to be the polynomial law if the coefficients of the appropriate operator $\mathbf{R}$ are the polynomials of $\mathbf{u}^{i}, \mathbf{v}^{i}$ and their derivatives by $x$. Using (3.4) one can check that the density of polynomial conservation law may be taken to be the polynomial without the loss of generality. Generally speaking the density of conservation law is defined up to the addition of the function $\mathbf{D}(\mathbf{f})$ for arbitrary function $\mathbf{f}$ of $\mathbf{u}^{i}, \mathbf{v}^{i}$ and their derivatives by $x$.

It's easy to check that

$$
\partial_{t}(\mathbf{R})+\mathbf{R}\left(\Lambda \mathbf{D}^{2}+\Phi\right)+\left(\Lambda \mathbf{D}^{2}+\boldsymbol{\Phi}^{\tau}\right) \mathbf{R}-\mathbf{S}=\sum_{i=0}^{m+2} \mathbf{M}_{i} \mathbf{D}^{i}
$$


with the matrices $\mathbf{M}_{i}$ of the form

$$
\mathbf{M}_{m+2}=\mathbf{R}_{m} \Lambda+\Lambda \mathbf{R}_{m}, \quad \mathbf{M}_{m+1}=\mathbf{R}_{m-1} \Lambda+\Lambda \mathbf{R}_{m-1}+2 \Lambda \mathbf{D}\left(\mathbf{R}_{m}\right)
$$

and so on. The operator equation (3.3) yields the system of $m+3$ matrix equations $\mathbf{M}_{i}=0$ with respect to the unknown $\mathbf{R}_{i}$. From the equation $\mathbf{M}_{m+2}=0$ we get $\mathbf{r}_{m}=0$, $\tilde{\mathbf{r}}_{m}=0$. The equation $\mathbf{M}_{m+2}=0$ is equivalent to $\tilde{\mathbf{r}}_{m-1}=0, \mathbf{D}\left(\mathbf{p}_{m}\right)=0, \mathbf{D}\left(\tilde{\mathbf{p}}_{m}\right)=0$. Let's denote $\mathbf{p}_{m}=\mathbf{Q}$, where $\mathbf{Q}$ is a constant matrix with elements $\mathbf{Q}_{i j}$. Then from (3.5) we get $\tilde{\mathbf{p}}_{m}=(-1)^{m} \mathbf{Q}^{\tau}$.

We may define the matrices $\mathbf{r}_{i-2}$ and $\tilde{\mathbf{r}}_{i-2}$ explicitly from each of the equations $\mathbf{M}_{i}=0$ with $i \geqq 2$. The matrices $\mathbf{p}_{i-1}$ and $\tilde{\mathbf{p}}_{i-1}$ are defined from the equations $\mathbf{D}\left(\mathbf{p}_{i-1}\right)=\eta_{i-1}, \mathbf{D}\left(\tilde{\mathbf{p}}_{i-1}\right)=\tilde{\eta}_{i-1}$, where $_{i-1}, \tilde{\eta}_{i-1}$ are already known matrices with the elements depending on $\mathbf{u}^{i}, \mathbf{v}^{i}$ and their derivatives by $x$. The technique for obtaining the solvability conditions for (3.3) is the same as that of the previous part. Applying the same procedure as in the case of symmetries we conclude that all the conservation laws are polynomials. So we may restrict our consideration to the conservation laws with homogeneous densities (for brevity we call them the homogeneous conservation laws). Similar to Propositions 2.1 and 2.2 we have the following statements.

Proposition 3.1. The density of homogeneous conservation law of the order $m=2 k$, $k \geqq 0$ has the form

$$
\varrho_{2 k}=\mathbf{Q}_{i j} \mathbf{u}_{k}^{i} \mathbf{v}_{k}^{j}+\kappa\left(\mathbf{u}, \mathbf{v}, \ldots, \mathbf{u}_{k-1}, \mathbf{v}_{k-1}\right),
$$

where $\kappa$ is the homogeneous polynomial with the weight $2 k+2$. The density of the homogeneous conservation law of the order $m=2 k+1, k \geqq 0$ has the form

$$
\varrho_{2 k+1}=\mathbf{Q}_{i j}\left(\mathbf{u}_{k+1}^{i} \mathbf{v}_{k}^{j}-\mathbf{u}_{k}^{i} \mathbf{v}_{k+1}^{j}\right)+\kappa\left(\mathbf{u}, \mathbf{v}, \ldots, \mathbf{u}_{k-1}, \mathbf{v}_{k-1}\right),
$$

where $\kappa$ is the homogeneous polynomial of the weight $2 k+3$.

Proposition 3.2. In order to have the conservation law of the order $m \geqq 1$ with the density (3.6) or (3.7) for the system (0.1) it is necessary to have the relationship

$$
\mathbf{Q}_{i r} \tilde{\mathbf{a}}_{j k m}^{r}-\mathbf{Q}_{r m} \mathbf{a}_{k j i}^{r}=0
$$

to be true for all indices $i, j, k, m$.

Remark 3.1. The condition (3.8) is not only necessary but also sufficient one for the existence of the conservation laws of first and second order. The homogeneous densities may be written in the form

$$
\begin{gathered}
\varrho_{1}=\mathbf{Q}_{i j}\left(\mathbf{u}^{i} \mathbf{v}_{x}^{j}-\mathbf{u}_{x}^{i} \mathbf{v}^{j}\right), \\
\varrho_{2}=\mathbf{Q}_{i j} \mathbf{u}_{x}^{i} \mathbf{v}_{x}^{j}-1 / 2 \mathbf{Q}_{r k} \mathbf{a}_{i m j}^{r} \mathbf{u}^{i} \mathbf{u}^{j} \mathbf{v}^{k} \mathbf{v}^{m} .
\end{gathered}
$$

In order for $(0.1)$ to have the conservation law of zero order of the system $(0.1)$ with the density $\varrho_{0}=\mathbf{Q}_{i j} \mathbf{u}^{i} \mathbf{v}^{j}$ it is necessary and sufficient that the relationship

$$
\mathbf{Q}_{i r} \tilde{\mathbf{a}}_{j k m}^{r}+\mathbf{Q}_{k r} \tilde{\mathbf{a}}_{j i m}^{r}-\mathbf{Q}_{r m} \mathbf{a}_{i j k}^{r}-\mathbf{Q}_{r j} \mathbf{a}_{i m k}^{r}=0
$$

holds for all indices $i, j, k, m$. The identity (3.11) is the consequence of (3.8), but the reverse statement is not true.

We shall give the algebraic interpretation for the relationship (3.8). The matrix $\mathbf{Q}$ defining the main part of the conservation law determines the bilinear form $\mathbf{Q}$ 
on the appropriate pair of vector spaces $(\mathbf{V}, \tilde{\mathbf{V}}): \mathbf{Q}\left(\mathbf{e}_{i}, \tilde{\mathbf{e}}_{j}\right)=\mathbf{Q}_{i j}$. Then (3.8) is equivalent to the identity

$$
\mathbf{Q}(\mathbf{x},\{\tilde{\mathbf{x}} \mathbf{y} \tilde{\mathbf{y}}\})=\mathbf{Q}(\{\mathbf{x} \tilde{\mathbf{y}} \mathbf{y}\}, \tilde{\mathbf{x}}),
$$

for all $\mathbf{x}, \mathbf{y} \in \mathbf{V}, \tilde{\mathbf{x}}, \tilde{\mathbf{y}} \in \tilde{\mathbf{V}}$. This means that $\mathbf{Q}$ is an invariant bilinear form. The following chain of equalities:

$$
\mathbf{Q}(\mathbf{x},\{\tilde{\mathbf{x}} \mathbf{y} \tilde{\mathbf{y}}\})=\mathbf{Q}(\mathbf{y},\{\tilde{\mathbf{x}} \mathbf{x} \tilde{\mathbf{y}}\})=\mathbf{Q}(\{\mathbf{x} \tilde{\mathbf{y}} \mathbf{y}\}, \tilde{\mathbf{x}})=\mathbf{Q}(\{\mathbf{x} \tilde{\mathbf{x}} \mathbf{y}\}, \tilde{\mathbf{y}})
$$

is a consequence of (3.12) and the symmetry of the multiplication with respect to the exterior arguments. Let $\mathbf{V}_{0}$ and $\widetilde{\mathbf{V}}_{0}$ be the linear subspaces in $\mathbf{V}$ and $\tilde{\mathbf{V}}$ such that $\mathbf{V}_{0} \stackrel{\text { def }}{=}\{\mathbf{x} \in \mathbf{V}, \mathbf{Q}(\mathbf{x}, \tilde{\mathbf{y}})=0$ for all $\tilde{\mathbf{y}} \in \tilde{\mathbf{V}}\}$,

$$
\tilde{\mathbf{V}}_{0} \stackrel{\text { def }}{=}\{\tilde{\mathbf{x}} \in \tilde{\mathbf{V}}, \mathbf{Q}(\mathbf{y}, \tilde{\mathbf{x}})=0 \text { for all } \mathbf{y} \in \mathbf{V}\} .
$$

Let's define $\operatorname{Ker} \mathbf{Q} \stackrel{\text { def }}{=}\left(\mathbf{V}_{0}, \tilde{\mathbf{V}}_{0}\right)$. From (3.13) we get that $\operatorname{Ker} \mathbf{Q}$ is an ideal.

The conservation law with the density defined principally by the invariant bilinear form will be called nondegenerate if $\operatorname{Ker} \mathbf{Q}=0$. Since $\operatorname{Ker} \mathbf{Q}$ is an ideal the following statement holds.

Proposition 3.3. Each system (0.1) possessing the degenerate conservation law of the order $m \geqq 1$ is reducible.

Obviously the system (0.1) may have the nondegenerate conservation law only if $N=M$.

Proposition 3.4. The system (0.1) with nondegenerate conservation law of the order $m \geqq 1$ is a hamiltonian system.

Proof. If the conservation law is nondegenerate if its matrix $\mathbf{Q}$ may be converted $P=\mathbf{Q}^{-1}$. The bilinear form $\mathbf{Q}$ satisfies the identity (3.8) providing the existence of the conservation law of the first order with the density (3.10) because of the Remark 3.1. This density plays the very thing for the role of hamiltonian if we take the antisymmetric matrix

$$
\mathbf{T}=\left(\begin{array}{cc}
0 & -\mathbf{P} \\
\mathbf{P} & 0
\end{array}\right)
$$

as a hamiltonian operator. It's easy to check up that because of (3.8) the system (0.1) may be represented as

$$
\mathbf{u}_{t}^{i}=-\mathbf{P}^{i r} \delta \varrho_{2} / \delta \mathbf{v}^{r}, \quad \mathbf{v}_{t}^{i}=\mathbf{P}^{i r} \delta \varrho_{2} / \delta \mathbf{u}^{r} .
$$

Let's turn back to Eq. (3.3). One can check that for the solvability of the equations $\mathbf{M}_{i}=0, i=m, \ldots, m-3$ it is necessary and sufficient to have the following equalities:

$$
\begin{aligned}
& \mathbf{Q}_{r i}\left(\mathbf{a}_{j k n}^{r} \mathbf{a}_{m s p}^{n}-\mathbf{a}_{m s n}^{r} \mathbf{a}_{j k p}^{n}-\mathbf{a}_{n s p}^{r} \mathbf{a}_{j k m}^{n}+\mathbf{a}_{m n p}^{r} \tilde{\mathbf{a}}_{k j s}^{n}\right)=0, \\
& \mathbf{Q}_{i r}\left(\tilde{\mathbf{a}}_{j k n}^{r} \tilde{\mathbf{a}}_{m s p}^{n}-\tilde{\mathbf{a}}_{m s n}^{r} \tilde{\mathbf{a}}_{j k p}^{n}-\tilde{\mathbf{a}}_{n s p}^{r} \tilde{\mathbf{a}}_{j k m}^{n}+\tilde{\mathbf{a}}_{m n p}^{r} \mathbf{a}_{k j s}^{n}\right)=0 .
\end{aligned}
$$

They provide the factor pair $(\mathbf{V}, \mathbf{V}) / \operatorname{Ker} \mathbf{Q}$ to be a Jordan pair. The relationships (3.16) were derived under the suggestion that the system has the conservation law of the order $m \geqq 4$. One can check that for the existence of the third order 
conservation law it is necessary and sufficient to have the conditions (3.8) and (3.16). Thus we have:

Theorem 3.1. The systems (0.1) with the nondegenerate conservation laws of the order $m \geqq 3$ are exhausted by the Jordan systems. The system (0.1) having the degenerate conservation law of the order $m \geqq 3$ is reducible, the factor-pair $(\mathbf{V}, \tilde{\mathbf{V}}) / \operatorname{Ker} \mathbf{Q}$ being correspondent to the Jordan system.

\section{Generalized Symmetries and Conservation Laws for Jordan Systems. Irreducible Jordan Systems}

In this chapter the questions dealing with the generalized symmetries and the conservation laws for Jordan systems are discussed. The formal recursion operator is constructed. The particular emphasis is made on the irreducible systems. The symmetric algebra and the infinite series of conservation laws for them are completely described.

Let's recall that the system $(0.1)$ is called integrable (see $[1,8]$ ) if the solution $\mathbf{L}$ of the following formal operator equation:

$$
\left[\Lambda \mathbf{D}^{2}+\boldsymbol{\Phi}, \mathbf{L}\right]-\partial_{t}(\mathbf{L})=0
$$

exists [compare with (2.3)]. Here $\mathbf{L}=\sum_{i=-\infty}^{n} \mathbf{L}_{i} \mathbf{D}^{i}$ is the formal series with the matrix coefficients $\mathbf{L}_{i}$ depending on $\mathbf{u}^{i}, \mathbf{v}^{i}$ and the finite number of their derivatives by $x$. The multiplication of the formal series is defined by

$$
\mathbf{D}^{k} \mathbf{D}^{m}=\mathbf{D}^{k+m}, \quad \mathbf{D}^{k} \mathbf{f}=\sum_{m=0}^{\infty} C_{m}^{k} \mathbf{D}^{m}(\mathbf{f}) \mathbf{D}^{k-m},
$$

for all $k, m$ where $C_{m}^{k}=k(k-1) \ldots(k-m+1) / m !$.

It is well known (see [1]) that nonlinear Schrödinger equation (0.3) is integrable. The following formal recursion operator is given for it the solution of (4.1),

$$
\mathbf{L}=\left(\begin{array}{cc}
\mathbf{D}+\mathbf{u} \mathbf{D}^{-1} \mathbf{v} & \mathbf{u} \mathbf{D}^{-1} \mathbf{u} \\
-\mathbf{v} \mathbf{D}^{-1} \mathbf{v} & -\mathbf{D}-\mathbf{v} \mathbf{D}^{-1} \mathbf{u}
\end{array}\right) .
$$

The analogous operator does exist for any Jordan system.

Theorem 4.1. Any Jordan system (0.1) is integrable and has its own formal recursion operator.

Proof. Here we give the explicit form of the formal recursion operator - the socalled "reduced" formal series

$$
\mathbf{L}=\left(\begin{array}{ll}
\mathbf{L}_{11} & \mathbf{L}_{12} \\
\mathbf{L}_{21} & \mathbf{L}_{22}
\end{array}\right)
$$

where blocks $\mathbf{L}_{i j}$ are given by

$$
\begin{array}{ll}
\mathbf{L}_{11}=\mathbf{I}_{N} \mathbf{D}+\mathbf{a}(j, k) \mathbf{u}^{j} \mathbf{D}^{-1} \mathbf{v}^{k}, & \mathbf{L}_{12}=\mathbf{A}(j, k) \mathbf{u}^{j} \mathbf{D}^{-1} \mathbf{u}^{k}, \\
\mathbf{L}_{22}=-\mathbf{I}_{M} \mathbf{D}-\tilde{\mathbf{a}}(j, k) \mathbf{v}^{j} \mathbf{D}^{-1} \mathbf{u}^{k}, & \mathbf{L}_{21}=-\tilde{\mathbf{A}}(j, k) \mathbf{v}^{j} \mathbf{D}^{-1} \mathbf{v}^{k} .
\end{array}
$$

We do not specify the way it has been obtained. Using (4.2) one can check up directly that (4.4) satisfies the operator equation (4.1) with respect to (1.10). 
By means of formal recursion operator (4.4) it seems to be possible to construct the symmetry for any Jordan system (0.1) of any higher order. Nowadays the author does not know the proof of this fact restricting himself with the statement of the hypothesis below.

Hypothesis. Each Jordan system (0.1) has the generalized symmetries of arbitrary order. The right-hand side of the homogeneous symmetry $\mathbf{u}_{\tau_{n}}^{i}=\mathbf{F}_{n}^{i}, \mathbf{u}_{\tau_{n}}^{i}=\mathbf{H}_{n}^{i}$ may be found by means of the recurrent formula

$$
\begin{aligned}
\mathbf{F}_{n}^{i} & =\mathbf{D}\left(\mathbf{F}_{n-1}^{i}\right)+\mathbf{u}^{j} \mathbf{D}^{-1}\left(\mathbf{a}_{j k m}^{i}\left(\mathbf{v}^{k} \mathbf{F}_{n-1}^{m}+\mathbf{u}^{m} \mathbf{H}_{n-1}^{k}\right)\right), \\
\mathbf{H}_{n}^{i} & =-\mathbf{D}\left(\mathbf{H}_{n-1}^{i}\right)-\mathbf{v}^{j} \mathbf{D}^{-1}\left(\tilde{\mathbf{a}}_{j k m}^{i}\left(\mathbf{u}^{k} \mathbf{H}_{n-1}^{m}+\mathbf{v}^{m} \mathbf{F}_{n-1}^{k}\right)\right) .
\end{aligned}
$$

where $\mathbf{F}_{1}^{i}=\mathbf{u}_{x}^{i}, \mathbf{H}_{1}^{i}=\mathbf{v}_{x}^{i}$.

It is proved directly by means of (4.6) that the symmetries of orders $n=3,4,5$ may be obtained. In order to prove that the right-hand side of (4.6) defines the symmetry of $n^{\text {th }}$ order it is sufficient to show that all components of $\mathbf{a}(j, k) \mathbf{u}^{j} \mathbf{v}^{k}$, $\tilde{\mathbf{a}}(j, k) \mathbf{v}^{j} \mathbf{u}^{k}$ are the densities for the conservation laws of the order $n-1$ and the right-hand sides of them are obtained by that recurrent formula.

Now let's proceed with conservation laws of Jordan systems. It is known (see [8]) that whenever the formal series satisfy Eq. (4.1) the function $\operatorname{tr}(\operatorname{res}(\mathbf{L}))$ with res $\left(\sum_{i=-\infty}^{n} \mathbf{s}_{i} \mathbf{D}^{i}\right) \stackrel{\text { def }}{=} \mathbf{s}_{-1}$ is the density for the conservation law (though it may be for the trivial one). We managed to construct the homogeneous formal series of the first order (4.4) with the matrix $\Lambda$ being the coefficient of $\mathbf{D}$. Along with it there exists the formal series of the first order with the unit coefficient of $\mathbf{D}$ satisfying Eq. (4.1). To obtain it one should find the square root of the series (4.4). Each product of entire powers of such series will also satisfy Eq. (4.1). Thus for any $m$ we have two homogeneous formal series $\mathbf{L}^{m}$ and $\tilde{\mathbf{L}}^{m}$, one of which begins with the matrix $\Lambda$ and the other begins with the unit matrix. One may check that

$$
\begin{gathered}
\operatorname{tr}\left(\operatorname{res}\left(\mathbf{L}^{2 k+1}\right)\right)=\left(\operatorname{tr}(\mathbf{a}(j, r))-\operatorname{tr}(\tilde{\mathbf{a}}(r, j)) \mathbf{u}_{k}^{j} \mathbf{v}_{k}^{r}+\kappa,\right. \\
\operatorname{tr}\left(\operatorname{res}\left(\tilde{\mathbf{L}}^{2 k+1}\right)\right)=\left(\operatorname{tr}(\mathbf{a}(j, r))+\operatorname{tr}(\tilde{\mathbf{a}}(r, j)) \mathbf{u}_{k}^{j} \mathbf{v}_{k}^{r}+\tilde{\kappa},\right. \\
\operatorname{tr}\left(\operatorname{res}\left(\mathbf{L}^{2 k+2}\right)\right)=\left(\operatorname{tr}(\mathbf{a}(j, r))+\operatorname{tr}(\tilde{\mathbf{a}}(r, j))\left(\mathbf{u}_{k+1}^{j} \mathbf{v}_{k}^{r}-\mathbf{u}_{k}^{j} \mathbf{v}_{k+1}^{r}\right)+\phi,\right. \\
\operatorname{tr}\left(\operatorname{res}\left(\tilde{\mathbf{L}}^{2 k+2}\right)\right)=\left(\operatorname{tr}(\mathbf{a}(j, r))-\operatorname{tr}(\tilde{\mathbf{a}}(r, j))\left(\mathbf{u}_{k+1}^{j} \mathbf{v}_{k}^{r}-\mathbf{u}_{k}^{j} \mathbf{v}_{k+1}^{r}\right)+\tilde{\phi},\right.
\end{gathered}
$$

where $\kappa, \tilde{\kappa}, \phi, \tilde{\phi}$ are the homogeneous polynomials of $\mathbf{u}^{i}, \mathbf{v}^{i}$ and their derivatives by $x$ with the orders lower or equal to $k-1$. Thus we obtain two series of conservation laws (usually they are called the canonic series). The densities for them are of the form (3.6) and (3.7) with $\mathbf{Q}_{i j}=\operatorname{tr}(\mathbf{a}(i, j))$ for the first seria and $\widetilde{\mathbf{Q}}_{i j}=\operatorname{tr}(\tilde{\mathbf{a}}(j, i))$. If at least one of these bilinear forms is nonzero then the system $(0.1)$ has the nontrivial conservation law of the order $m$.

Note that bilinear forms defining the quadratic parts of densities for canonic conservation laws are canonic also from an algebraic point of view. Let the linear operator $\mathbf{M}(\mathbf{x}, \tilde{\mathbf{y}}): \mathbf{V} \rightarrow \mathbf{V}$ be defined by $\mathbf{M}(\mathbf{x}, \tilde{\mathbf{y}})(\mathbf{z})=\{\mathbf{x} \tilde{\mathbf{y}} \mathbf{z}\}$. Then the bilinear form $\mathbf{Q}(\mathbf{x}, \tilde{\mathbf{y}})=\operatorname{tr}(\mathbf{M}(\mathbf{x}, \tilde{\mathbf{y}}))$ is invariant, i.e. the identity (3.12) holds for it. Analogously one can define the second invariant form $\tilde{Q}(\mathbf{x}, \tilde{\mathbf{y}})=\operatorname{tr}(\tilde{\mathbf{M}}(\tilde{\mathbf{y}}, \mathbf{x}))$, where the linear operator $\tilde{\mathbf{M}}(\tilde{\mathbf{y}}, \mathbf{x}): \tilde{\mathbf{V}} \rightarrow \tilde{\mathbf{V}}$ is defined by $\tilde{\mathbf{M}}(\tilde{\mathbf{y}}, \mathbf{x})(\tilde{\mathbf{z}})=\{\tilde{\mathbf{y}} \mathbf{x} \tilde{\mathbf{z}}\}$.

The above considerations leads us to the following statement. 
Theorem 4.2. The system (0.1) corresponding to the Jordan pair which has at least one nonzero bilinear form $\operatorname{tr}(\mathbf{M}(\mathbf{x}, \tilde{\mathbf{y}}))$ or $\operatorname{tr}(\tilde{\mathbf{M}}(\tilde{\mathbf{x}}, \mathbf{y}))$ has the infinite series of nontrivial canonic conservation laws.

Let's consider in more detail the irreducible Jordan systems (0.1). First of all note that the existence of classification for simple Jordan pairs over $\mathbb{C}$ enables us to classify all the irreducible generalized Schrödinger equations and then describe them constructively. Now we present the list of simple Jordan pairs which consists of four infinite series and two special Jordan pairs. In each case $\mathbf{V}$ and $\tilde{\mathbf{V}}$ have the same dimensions as vector spaces, i.e. Jordan pairs correspond to the irreducible systems $(0.1)$ of $2 N$ equations.

Every simple Jordan pair over $\mathbb{C}$ is contained in the following list (see [5]):

$\mathrm{I}_{p, q} \cdot\left(\mathbf{M}_{p, q}(\mathbb{C}), \mathbf{M}_{p, q}(\mathbb{C})\right), p \times q$ matrices over $\mathbb{C}$, where $1 \leqq p \leqq q$. The dimension of corresponding Jordan system $(0.1)$ is $p q \times p q$.

$\mathrm{II}_{n} \cdot\left(\mathbf{A}_{n}(\mathbb{C}), \mathbf{A}_{n}(\mathbb{C})\right)$, alternating $n \times n$ matrices over $\mathbb{C}$, where $n \geqq 5$. The dimension of corresponding Jordan system $(0.1)$ is $n(n+1) / 2+n(n+1) / 2$.

$\mathrm{III}_{n} .\left(\mathbf{H}_{n}(\mathbb{C}), \mathbf{H}_{n}(\mathbb{C})\right)$, symmetric $n \times n$ matrices over $\mathbb{C}$, where $n \geqq 2$. The dimension of corresponding Jordan system $(0.1)$ is $(n(n+1) / 2+n(n+1) / 2$.

In these three cases, the Jordan pair structure is given by

$$
\{\mathbf{x y z}\}=\mathbf{x}^{t} \mathbf{y} \mathbf{z}+\mathbf{z}^{t} \mathbf{y} \mathbf{x} .
$$

$\operatorname{IV}_{n} \cdot\left(\mathbb{C}^{n}, \mathbb{C}^{n}\right)$, where $n \geqq 4$, the Jordan pair structure is given by standard quadratic form on $\mathbb{C}^{n}$. The dimension of corresponding Jordan system $(0.1)$ is $n+n$.

V. $\left(\mathbf{M}_{1,2}(\mathbf{C}), \mathbf{M}_{1,2}\left(\mathbf{C}^{o p}\right)\right), 1 \times 2$ matrices over the Cayley algebra $\mathbf{C}$ over $\mathbb{C}$. The dimension of the corresponding Jordan system $(0.1)$ is $16+16$.

VI. $\left(\mathbf{H}_{3}(\mathbf{C}), \mathbf{H}_{3}(\mathbf{C})\right)$, the Jordan pair associated with the exceptional Jordan algebra of $3 \times 3$ Hermitian matrices over $\mathbf{C}$. The dimension of the corresponding Jordan system $(0.1)$ is $27+27$.

The following statement provides the full description of the structure of the set of local conservation laws and generalized symmetries of the irreducible systems (0.1).

Theorem 4.3. The homogeneous local conservation laws of the order $m \geqq 1$ of the irreducible Jordan systems (0.1) are exhausted by the canonical series of nondegenerate conservation laws with the densities of the form

$$
\begin{aligned}
\varrho_{2 k} & =\operatorname{tr}(a(i, j)) \mathbf{u}_{k}^{i} \mathbf{v}_{k}^{j}+\kappa\left(\mathbf{u}, \mathbf{v}, \ldots, \mathbf{u}_{k-1}, \mathbf{v}_{k-1}\right), \\
\varrho_{2 k+1} & =\operatorname{tr}(a(i, j))\left(\mathbf{u}_{k+1}^{i} \mathbf{v}_{k}^{j}-\mathbf{u}_{k}^{i} \mathbf{v}_{k+1}^{j}\right)+\kappa\left(\mathbf{u}, \mathbf{v}, \ldots, \mathbf{u}_{k-1}, \mathbf{v}_{k-1}\right) .
\end{aligned}
$$

The homogeneous generalized symmetries are exhausted by the symmetries written in the hamiltonian form

$$
\mathbf{u}_{\tau_{n}}^{i}=-\mathbf{P}^{i r} \delta \varrho_{n} / \delta \mathbf{v}^{r}, \quad \mathbf{v}_{\tau_{n}}^{i}=\mathbf{P}^{i r} \delta \varrho_{n} / \delta \mathbf{u}^{r},
$$

where $\mathbf{P}^{\text {ir }}$ are elements of the matrix $\mathbf{P}$, inverse with respect to the matrix of invariant bilinear form $\mathbf{Q}_{i j}=\operatorname{tr}(a(i, j))$ and $\varrho_{n}$ are the densities (4.7).

Proof. For each simple Jordan pair the bilinear forms $\operatorname{tr}(\mathbf{M}(\mathbf{x}, \tilde{\mathbf{y}}))$ and $\operatorname{tr}(\tilde{\mathbf{M}}(\tilde{\mathbf{x}}, \mathbf{y}))$ mentioned above are nondegenerate and proportional each other (see [5]). Therefore, for the irreducible Jordan systems (0.1) both canonical seria coincide 
and their densities has the form (4.7). Now we have only to check up the absence of the homogeneous conservation laws of the order $m \geqq 1$ different from the canonical ones. In order to do this one may show that the invariant bilinear form $\mathbf{Q}(\mathbf{x}, \tilde{\mathbf{y}})$ defining the quadratic part of the density for any conservation law should be proportional to the canonical invariant form $\operatorname{tr}(\mathbf{M}(\mathbf{x}, \tilde{\mathbf{y}}))$. If it is not the case then there exists the conservation law defined by the degenerate but nonzero bilinear form. This leads to the contradiction with Proposition 3.3 and the irreducibility of the systems $(0.1)$.

Thanks to the presence of the nondegenerate conservation law of the second order each irreducible Jordan system $(0.1)$ is a hamiltonian one and can be written as (3.15). It is well known (see for example [9]) that if $\varrho$ is the density of conservation law for the system (3.15) then

$$
\mathbf{u}_{\tau}^{i}=-\mathbf{P}^{i r} \delta \varrho / \delta \mathbf{v}^{r},, \quad \mathbf{v}_{\tau}^{i}=\mathbf{P}^{i r} \delta \varrho / \delta \mathbf{u}^{r}
$$

is the symmetry of that system. We have only to show that irreducible systems can't have the homogeneous generalized symmetries different from (4.8). The presence of the additional generalized symmetry leads to the existence of the degenerate linear operator $\mathbf{K}$ (or $\widetilde{\mathbf{K}}$ ) satisfying the equalities (2.20), (2.21) which in turn contradicts to irreducibility.

For the conclusion note that all irreducible generalized Schrödinger equations perhaps may be obtained by means of the construction from [4], too.

Acknowledgements. The author is grateful to V. V. Sokolov and A. B. Shabat for useful discussions and remarks.

\section{References}

1. Mikhailov, A.V., Shabat, A.B., Yamilov, R.I.: Symmetry approach to the classification of nonlinear equations. USP. Mat. Nauk 42, No. 4, 3-53 (1987)

2. Ablowitz, M.J.: Lectures on the inverse scattering transform. Stud. Appl. Math. 108, No. 1, 17-94 (1978)

3. Jiber, A.V.: $N$-wave equation and the system of nonlinear Schrödinger equations from the group theoretical point of view. Teor. Mat. Fiz. 52, No. 3, 405-413 (1982)

4. Fordy, A., Kulish, P.: Nonlinear Schrödinger equations and simple Lie algebras. Commun. Math. Phys. 89, 427-443 (1983)

5. Loos, O.: Jordan pairs. Lect. Notes Math., vol. 460, p. 218. Berlin, Heidelberg, New York: Springer 1975

6. Bachturin, Yu.A., Slin'ko, A.M., Shestakov, I.P.: Nonassociative rings. Sov. Probl. Matem. VINITI, 18, 3-72 (1981)

7. Loos, O.: A structure theory of Jordan pairs. Bull. Am. Math. Soc. 80, No. 1, 67-71 (1974)

8. Mikhailov, A.V., Sokolov, V.V., Shabat, A.B.: Symmetry approach to the classification of integrable equations. In: What is integrability? Berlin, Heidelberg, New York: Springer 1991

9. Olver, P.: Applications of Lie groups to differential equations. Berlin, Heidelberg, New York: Springer 1986 
